\title{
Isometric contraction of the abductor digiti minimi muscle in man
}

\author{
DAVID BURKE, NEVELL F. SKUSE, AND A. KEITH LETHLEAN \\ From the Unit of Clinical Neurophysiology, Division of Neurology, \\ Prince Henry Hospital, Little Bay, N.S.W. 2036, Australia
}

SYNOPSIS Isometric contraction of the abductor digiti minimi muscle (ADM) has been studied in six normal subjects. Twitch contraction times of ADM ranged from 60 to $68 \mathrm{~ms}$ and twitch torque ranged from $2 \cdot 33$ to $6.24 \times 10^{-3} \mathrm{Nm}$. In three subjects torque declined by an average of $31 \%$ after tetanization at $50 \mathrm{~Hz}$ for 30 seconds but there was no similar diminution in the evoked muscle action potential suggesting that the fatigue arose from intrinsic muscular mechanisms. A marked decline in tetanic torque occurred with continued tetanization in two subjects for a total of five minutes, but this change was accompanied by a decrease in the muscle action potential. In six subjects threshold stimulation to the ulnar nerve at the wrist and to various sites over the motor point of ADM allowed 55 threshold twitch contractions to be identified after averaging. A unimodal range of contraction times ranging from $40-100 \mathrm{~ms}$ was found and this was confirmed by additional experiments in two subjects in whom 30 threshold twitch contractions were identified using a needle electrode to stimulate various sites in the motor point. Tetanization at $50 \mathrm{~Hz}$ was performed using threshold stimulus levels. Nine threshold tetanic contractions were evoked in two subjects. In eight tetanic torque progressively fatigued to between 14 and $20 \%$ within $60-90$ seconds, but, in one tetanic contraction, torque proved relatively fatigue resistant. These results suggest that there is a homogeneous group of motor units in ADM (with respect to contraction time) and that this group contains what are probable fast twitch fatigue sensitive and fatigue resistant motor units. No evidence of a distinct group of slow twitch units was found.

The documentation of motor unit properties in man remains in its infancy. Isometric twitch contractions have been elicited in the adductor pollicis muscle (Merton, 1954; Desmedt, 1958; Slomić et al., 1968), in frontalis, first dorsal interosseous, lateral gastrocnemius, and extensor digitorum brevis (McComas and Thomas, 1968), and in extensor hallucis brevis (Sica and McComas, 1971) but activation of an entire muscle provides little data about its constituent motor units. In biopsy specimens of different human muscles fast and slow twitch fibres have been found (Brust and Cosla, 1967; Eberstein and Goodgold, 1968) but in vivo studies of individual motor units have been few. In soleus, gastrocnemius, tibialis anterior, and biceps and triceps brachii, Buchthal and Schmalbruch (1970) demonstrated a correlation between contraction time and fibre type, and in extensor hallucis brevis (EHB) Sica and McComas (1971) were able to distinguish two groups of motor units (fast and slow twitch units) by averaging the mechanical response to threshold stimulation of the motor nerve. Perhaps the most sophisticated in vivo technique has been that of MilnerBrown et al. (1973a, b, c) who have determined the contractile properties of individual motor units in the first dorsal interosseous muscle (IDI) of human subjects during voluntary contraction.

This study presents preliminary observations on the isometric properties of the abductor digiti minimi muscle (ADM) in human subjects and documents the method used for studying the contractile properties of ADM. The total muscle twitch response, the response to tetanization, and the properties of individual motor units activated by threshold stimulation have been determined in six normal subjects. These data 


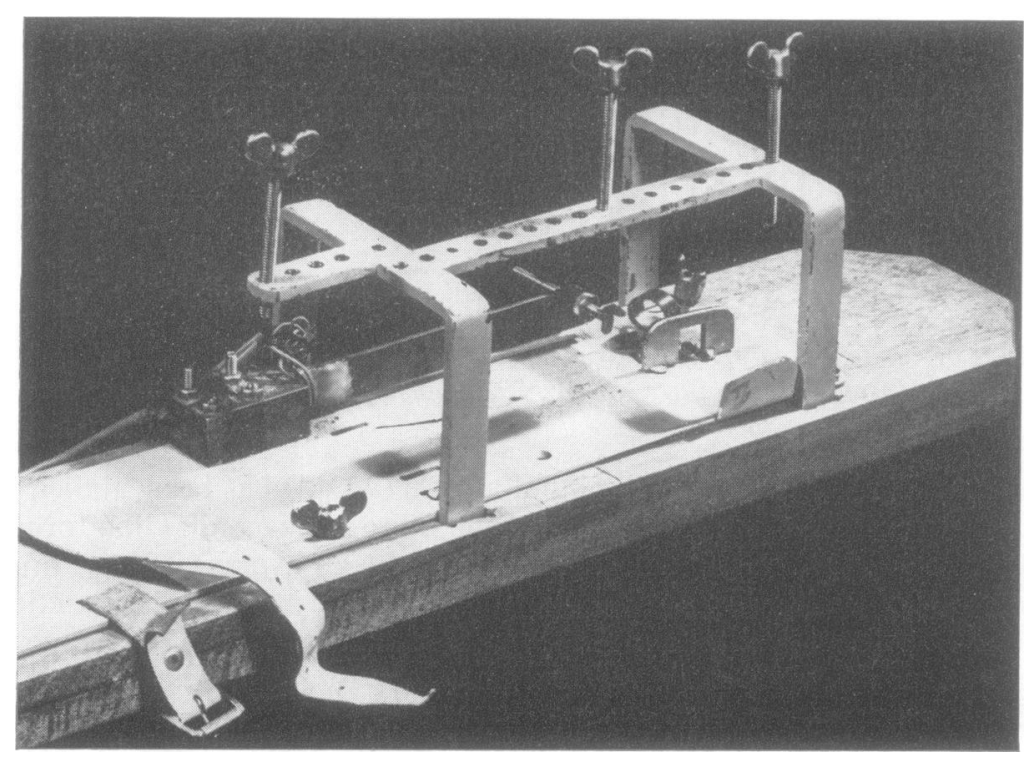

FIG. 1. The frame used for recording isometric contractions of ADM. For simplicity the three padded compressive metal plates which fix the arm and hand are not shown. These plates are screwed down onto the limb from the superstructure by the three screws illustrated, the positions of which can be altered.

will serve as control observations for a subsequent paper on paramyotonia congenita (Burke et al., 1974).

\section{METHODS}

The data to be presented were obtained from 18 experiments in six normal subjects aged 22 to 45 years. Two of the authors were studied in greater detail because of availability and because intramuscular recordings and tetanization as used in some of the studies were not readily acceptable to volunteer subjects.

The isometric contraction of ADM was studied with the right hand rigidly immobilized in a metal frame designed for use in myasthenic patients by Dr George Preswick (Fig. 1). Subjects lay supine on a couch with the right arm abducted at the shoulder, extended at the elbow, and immobilized by a leather strap fixed around the upper forearm and by three padded metal plates which were screwed down from the overhead supporting frame. Two of these compressive plates fixed the limb at wrist and palm levels, while a third plate immobilized the index, middle, and ring fingers, which were taped together and which were additionally isolated from the fifth finger by an adjustable vertical plate. Flexion movements of the fifth finger in the vertical plane were prevented by a retaining ring over the distal end of the middle phalanx. The retaining ring was free to move laterally along a horizontal slot in the base- plate of the frame so that abduction movements the fifth finger were unimpeded. Such lateral (or.abduction) movement was then restricted by a teb- $c$ sion transmitting rod with an ' $L$ ' shaped end whigh was fitted over the proximal phalanx at the met $\overrightarrow{0}$ carpophalangeal joint. The other end was fixed two nuts into a slot in a mild steel bar of length 15 . $\mathrm{cm}$ such that the length of the tension transmitting rod could be altered where necessary. A four-arm strain gauge bridge was constructed by bonding four Phillips etched foil strain gauges (Type PR 9831) to $\frac{\square}{\varnothing}$ the steel bar and this bridge was energized by $a \propto$ Tektronix carrier amplitier type 3C66. The length of $\overrightarrow{\overrightarrow{0}}$ the tension transmitting rod was adjusted to impart slight stretch to ADM, thus 'pretensioning' the strain gauge bridge. Compliance of the steel bar was? low, such that a $1 \mathrm{~kg}$ weight attached to the end of the bar produced a displacement of less than $1 \mathrm{~mm}$. Recording were therefore virtually isometric. The natural (resonant) frequency of the metal arm of the strain gauge was $100 \mathrm{~Hz}$ in the unloaded state.

Stimuli of $0.2 \mathrm{~ms}$ duration were delivered by two

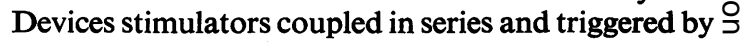
a Devices Digitimer. For studies of the total muscle $\rightarrow$ twitch characteristics supramaximal stimuli were delivered by surface electrodes $2 \mathrm{~cm}$ apart taped to $\mathrm{N}$ the wrist over the ulnar nerve. Stimulus voltage was increased for tetanization to exceed maximal levels 0 by approximately $50 \%$, thus hopefully avoiding the $\omega$ effects of the excitability changes described by Bergmans (1970). For studies of threshold motoro 
unit twitches, threshold stimuli were also delivered to the motor point of ADM using a pencil-shaped monopolar stimulating electrode. For this purpose, this electrode was held in a stereotactic micromanipulator so that changes in position of the point of stimulation could be readily produced.

The output of the strain gauge bridge was displayed on a Tektronix 564 storage oscilloscope and photographed when necessary with a Polaroid oscilloscope camera. In some experiments the electromyogram (EMG) of ADM was recorded by surface electrodes taped over ADM, one directly over the motor point and the other over the metacarpophalangeal joint. The recorded muscle action potential was amplified by a Tektronix 122 low level preamplifier (high and low frequency filters of 1000 $\mathrm{Hz}$ and $0.2 \mathrm{~Hz}$ respectively) and displayed on a second channel of the storage oscilloscope.

For studies of threshold motor units, the strain gauge output was monitored on the storage oscilloscope and fed into a ND801 Enhancetron 1024 in which between 100 and 500 consecutive contractions were averaged. The read-out of the Enhancetron was displayed on a second oscilloscope and photographed with a Polaroid oscilloscope camera. Because arterial pulsation often produced a force which exceeded that produced by contraction of threshold motor units, threshold stimulation was performed during arterial occlusion produced by a sphygmomanometer cuff inflated to $200 \mathrm{mmHg}$ and placed above the elbow. Efforts were made to minimize the duration of circulatory arrest to less than five minutes, since Buchthal and Schmalbruch (1970) have demonstrated that prolonged hypoxia eliminates slower contraction times within a muscle, thus shifting the peak of the spectrum to fast contraction times. Threshold stimuli were therefore delivered at the rate of $2 \mathrm{~Hz}$. Such low frequency stimulation evokes the 'positive staircase' phenomenon, but with the technique used the slight inaccuracy produced was constant for any one patient, and this factor has therefore been ignored (see Results section for further justification).

Experiments were all performed in an airconditioned laboratory. Skin temperature was measured over the belly of ADM in some experiments using an Ellab electronic thermometer and surface temperatures of $33-35^{\circ} \mathrm{C}$ were maintained.

BRIDGE CALIBRATION The output of the strain gauge bridge was calibrated in arbitrary units and was found to be linear over the entire working range, such that 1 unit was equivalent to $0.476 \mathrm{~g}$ weight or 4.66 milliNewtons $(\mathrm{mN})$. More accurately, the measurements of contractile strength of ADM should be expressed not as force (in grammes weight or in $\mathrm{mN}$ ) but as torque (in Newton-metres) because the output was measured at a point on the proximal phalanx $1 \mathrm{~cm}$ distal to the insertion of ADM. Thus a bridge output of 1 unit may be considered equivalent to a torque of $4.66 \times 10^{-5}$ Newton-metres $(\mathrm{Nm})$. Since other authors have not expressed contractile strength in units of torque, the available data on other muscles cannot be compared with those to be presented for ADM.

\section{RESULTS}

MECHANICAL PROPERTIES OF ABDUCTOR DIGITI MINIMI The torque produced by supramaximal contraction of ADM ranged from $2.33 \times 10^{-3}$ $\mathrm{Nm}$ to $6.24 \times 10^{-3} \mathrm{Nm}$ (mean $3.77 \times 10^{-3} \mathrm{Nm}$ ).

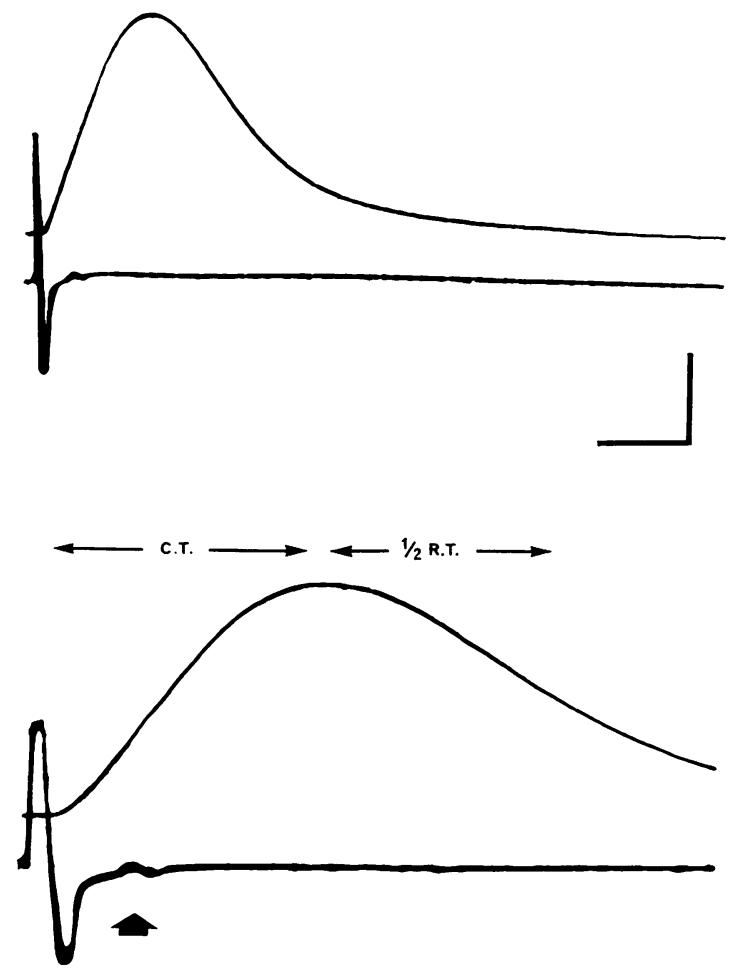

FIG. 2. The isometric twitch contraction of $A D M$. Twitch torque and the evoked muscle action potential are shown at two different sweep speeds. Three contractions are superimposed on each occasion. Note the small $F$ wave, indicated by the arrow. Contraction time (C.T.) and half relaxation time ( $\frac{1}{2}$ R.T.) are as indicated. Calibration: vertical: $2.33 \times 10^{-3} \mathrm{Nm}$, $2 \mathrm{mV}$; horizontal: $50 \mathrm{~ms}$ and $20 \mathrm{~ms}$ for upper and lower traces respectively. 
The contraction was always of smooth contour (Fig. 2) provided that the retaining ring fitted firmly around the finger. The twitch contraction time, measured from the onset to the peak of muscle contraction, was 60-68 ms (mean $63 \mathrm{~ms}$ ). The half-relaxation time, measured from peak contraction to the point at which contractile torque had decayed to $50 \%$ of the peak level, ranged from 60 to $80 \mathrm{~ms}$ (mean $71 \mathrm{~ms}$ ).

A small $\mathrm{F}$ wave was commonly recorded in the EMG in response to supramaximal stimulation, the amplitude of this wave being less than $6 \%$ of the amplitude of the direct motor $(\mathrm{M})$ response. Conceivably, the presence of the $F$ wave could have prolonged the relaxation phase slightly, but in the adductor pollicis Slomić et al. (1968) have shown that this factor is negligible and it has therefore been ignored.

Repetitive stimulation and tetanization were performed in three subjects using supramaximal stimulus levels. Repetitive stimulation at $3 \mathrm{~Hz}$ for two minutes induced the 'positive staircase' phenomenon as described by Desmedt and Hainaut (1968), Slomić et al. (1968), and Desmedt et al. (1973). Twitch torque progressively increased during stimulation by $33-45 \%$. Contraction time decreased by $8-10 \%$ within the first 10-20 stimuli and thereafter remained relatively constant. The rate of development of twitch torque was therefore greatly increased. There was no concomitant change in the evoked muscle action potential so that these results are consistent with those reported by other authors.

The frequency at which a fused tetanus was produced was $25-30 \mathrm{~Hz}$. Peak tetanic force was $4.66-6.9 \times 10^{-2} \mathrm{Nm}$ and the twitch-tetanus ratio was 0.09 . The tetanic contraction was well maintained over short periods (up to $10 \mathrm{~s}$ ) but a gradual decline in peak torque occurred with prolonged tetanization (Fig. 3a) so that in three normal subjects (seven observations) the contraction level had fallen by an average of $31 \%$ after $30 \mathrm{~s}$. There was no concomitant decrease in the evoked muscle action potential to each stimulus. The amplitude of the negative phase of the action potential was virtually unchanged but the area of the negative phase as measured by planimetry was actually increased due to widening and slurring of the action potential (Fig. 3b). It may be concluded that the $30 \%$ decline in tetanic torque was due to intrinsic muscular

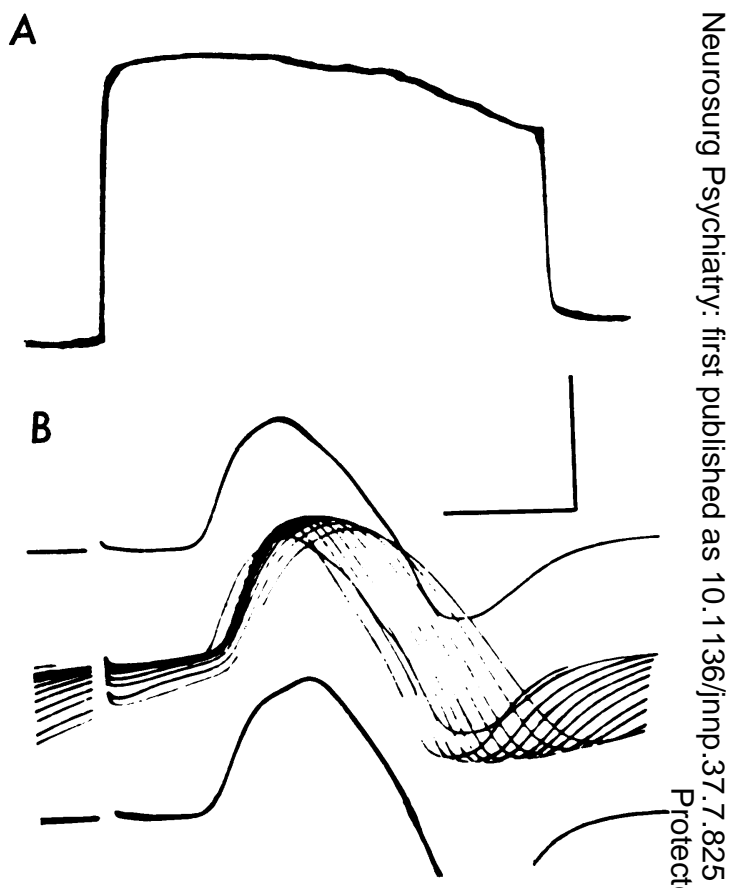

FIG. 3. Tetanization at $50 \mathrm{~Hz}$ for $30 \mathrm{~s}$ at supr赵음 maximal voltage. A. The mechanical response de creases by $30-35 \%$ within $30 \mathrm{~s}$. B. The evoked muscte $\subseteq$ action potential. In each of the upper and lower traces three isolated contractions are superimposed before and $\vec{\omega}$ after tetanization respectively. In the middle trace th change with tetanization is seen. The potential evoked by the first stimulus of the tetanus is shown and every 3 s throughout the tetanus a further potential has been recorded. Note the progressive widening of the potential without loss of amplitude of the negative phase. The area of the negative phase increases progressively. Calibrations: vertical: $2.33 \times 10^{-2} \mathrm{Nm}$ for A, $3 \mathrm{mV}$. for B; horizontal: $10 \mathrm{~s}$ for A, 4 ms for B.

mechanisms rather than to fatigue at the myoneural junction.

In two subjects tetanization was continued for five minutes. Tetanic contraction was again well $₹$

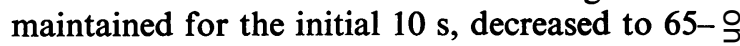
$70 \%$ by $30 \mathrm{~s}$, but decreased further to $25 \%$ over $D$ the ensuing $90 \mathrm{~s}$. Thereafter a plateau level was maintained for the final three minutes with only $\mathrm{N}^{\circ}$ slight further decrease (Fig. 4). As measured by planimetry, the area of the negative phase of the evoked muscle action potential was again larger after tetanization for $30 \mathrm{~s}$ due to slurring of the 


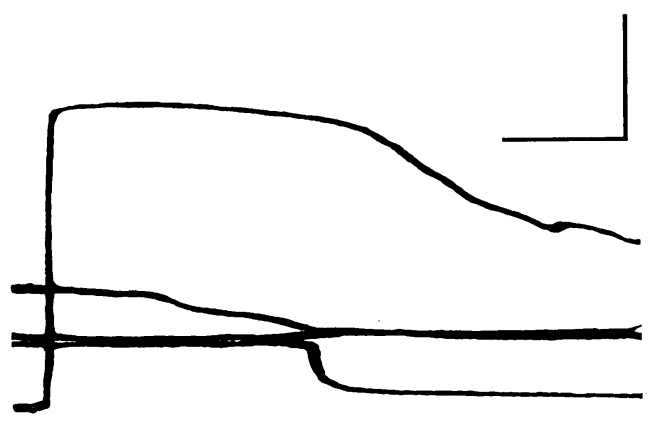

FIG. 4. Tetanic torque during prolonged tetanization at $50 \mathrm{~Hz}$ at supramaximal voltage for five minutes: each oscilloscope sweep lasts $50 \mathrm{~s}$. The sweep was triggered four times during tetanization to record the start, the finish, and two different stages during tetanization, $60 \mathrm{~s}$ being allowed to elapse between successive sweeps. Maximal fatigue occurs early between 30 and $90 \mathrm{~s}$ and thereafter only slight further fatigue occurred. Calibrations: vertical: $2.33 \times 10^{-2}$ $\mathrm{Nm}$; horizontal: $10 \mathrm{~s}$.

potential, but after tetanization for $60 \mathrm{~s}$ the amplitude of the negative phase decreased greatly so that the area of the negative phase was $44 \%$ of its initial value. A slight further decrease occurred with continued stimulation. Thus only a part of the fatigue produced by prolonged tetanization can be attributed with certainty to intrinsic muscular mechanisms.

MOTOR UNIT COUNTS An attempt was made to estimate the number of motor units in ADM by determining the average EMG increment produced by progressively larger stimulus levels, as originally described for extensor digitorum brevis (EDB) by McComas et al. (1971) and as recently applied to the thenar muscles by Brown (1972, 1973). Such studies suggested that ADM contained between 100 and 150 motor units. However, even with careful placement of surface electrodes, it proved impossible to record selectively from ADM, although the pick-up from the other hypothenar muscles could be reduced to less than one-third the voltage recorded from ADM. The motor axons of lowest threshold in the ulnar nerve at the wrist were found to innervate hypothenar muscles other than ADM in at least two subjects, so that low voltage EMG activity was picked up by the electrodes over
ADM but no muscle twitch was recorded by the strain gauge.

In Brown's experiments the surface electrodes were positioned over the abductor pollicis brevis and the calculated motor unit counts were considered to be estimates of motor units in the thenar muscle group. In our experiments, while surface electrodes over ADM picked up the activity of other hypothenar muscles (flexor digiti quinti brevis and opponens digiti quinti), such activity always appeared somewhat attenuated. EMG increments due to recruitment of motor units to these muscles would appear falsely small, and, as a result, discrepancies in motor unit counts could arise. Moreover, it cannot be assumed that the action potentials evoked by activation of motor units from the three hypothenar muscles would sum algebraically, as required by the technique of McComas et al. (1971), who pointed out that their technique was not reliable for muscles other than extensor digitorum brevis of the foot.

Estimation of the number of motor units in ADM was also attempted by dividing the torque recorded on supramaximal stimulation by the average size of torque increments. With supraliminal stimuli a number of units commonly presented with similar electrical thresholds, and only with near-threshold stimuli could individual units be activated. Such estimates suggested between 50 and 100 motor units for ADM but this figure is likely to be an underestimate because difficulty was experienced in distinguishing individual torque increments. However, as shown in the next section, single motor units produced a torque of generally less than $4.66 \times 10^{-5} \mathrm{Nm}$ and never more than $9.32 \times 10^{-5} \mathrm{Nm}$, and division of the total muscle twitch torque by these values provides a motor unit estimate of similar magnitude to the calculated estimate.

THRESHOLD MOTOR UNITS With liminal stimuli to the ulnar nerve at the wrist or to the motor point of ADM, motor unit twitch contractions could be identified which behaved in an 'all-ornone' manner and which therefore conform to the criteria of single motor unit contractions. The surface anatomy of the motor point was mapped in six subjects using monopolar percutaneous stimulation. The motor point appeared to be shaped like a narrow-based isosceles 
triangle or like the figure ' $Y$ ', oriented longitudinally with the base of the triangle or the open arms of the $U$ pointing distally. Considering the distortion produced by percutaneous stimulation, these results are in reasonable agreement with the anatomical studies of motor point anatomy detailed by Desmedt (1958) who found an elongated ' $U$ '-shaped motor point.

Threshold contractions, whether induced by percutaneous stimulation at the wrist or over the motor point, produced a torque of usually less than $4.66 \times 10^{-5} \mathrm{Nm}$ and always less than $9.32 \times 10^{-5} \mathrm{Nm}$. To determine the time course of single motor unit twitches, 100-500 consecutive threshold stimuli at $2 \mathrm{~Hz}$ were summed by a fixed programme averaging computer during arterial occlusion. Care had to be taken at each stimulus site to ensure that a stimulus which appeared threshold for only one motor unit during single stimuli did not activate more than one unit during repetitive stimulation at $2 \mathrm{~Hz}$. At times the stimulus level had to be set so that the threshold unit was not always activated. It was therefore not possible to determine the twitch torque of threshold units accurately by feeding a calibration pulse into the averaging input (as done by Sica and McComas, 1971), since with such 'all-or-none' behaviour the pulse would have been summed at times when no contraction was recorded.

Low frequency repetitive stimulation even at threshold levels induced the 'positive staircase' phenomenon in the activated motor units, with resultant increase in twitch torque and decreases in contraction and half-relaxation times. Changes in twitch torque are relatively unimportant to the present study, since absolute values for twitch torque cannot be given. Decreases in twitch times are more relevant but these changes occur quite rapidly, generally in the first 10-20 stimuli, after which a plateau level is maintained (Desmedt et al., 1973; see above). Care was taken in the present experiments to start repetitive stimulation $20-40$ s before averaging, and during this period stimulus levels were determined and computer balance levels were adjusted. These procedures had to be carried out before each averaging run so that the plateau level of twitch times was reached on each occasion before averaging commenced. The values obtained are therefore all slightly lower

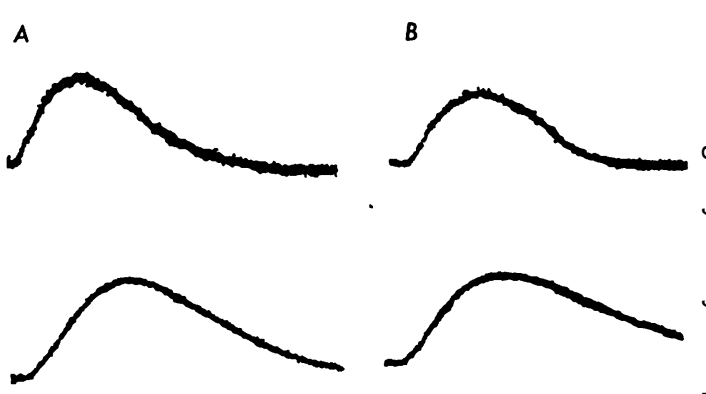

FIG. 5. Threshold motor unit twitches. A. Surface $\frac{\overline{\bar{O}}}{\overline{\mathrm{g}}}$ stimulation at the motor point. Upper trace: averaged $\stackrel{\text { क }}{\vec{\Phi}}$ 200 sweeps, contraction time $46 \mathrm{~ms}$. Lower trace: averaged 250 sweeps, contraction time $76 \mathrm{~ms}$. B.ळ Intramuscular stimulation at the motor point. Upper trace: averaged 200 sweeps, contraction time $54 \mathrm{~ms}$. Lower trace: averaged 200 sweeps, contraction time $\vec{\omega}$ $74 \mathrm{~ms}$. In both A and B duration of trace is $250 \mathrm{~ms}$.

than those which would have been recorded wi single stimuli.

A total of 55 threshold contractions was re을 corded in six subjects, and the averaged twitchesof two threshold units are shown in Fig. 5a. TRe twitch contraction times of these 55 threshofid contractions are shown in Fig. 6a. A unimod嘀 distribution of these times is seen, unlike the bit:modal distribution reported for EHB by Sica and McComas (1971). The failure to find a bimodal distribution of contraction times (1) could result from masking of such a distribution due to pooling of data from different subjects; (2) could be due to discrepancies in technique such that some units were overrepresented, being of $\frac{3}{5}$ lowest threshold at a number of stimulation sites; or (3) could represent the true distribution of twitch times within this muscle. The first possibility seems unlikely, since a bimodal dis- $\frac{0}{0}$ tribution was not apparent in the data for individual subjects. The second possibility also seems unlikely, since with a discrete stimulating electrode point a number of well-separated 윽 stimulation sites can be found on the relatively $\frac{D}{2}$ large surface area of the motor point, and since a spectrum of contraction times was recorded in $N$ each subject. Nevertheless it cannot be denied $N$ that some units may have been represented more N than once in the histogram.

In an attempt to validate the unimodal dis- 


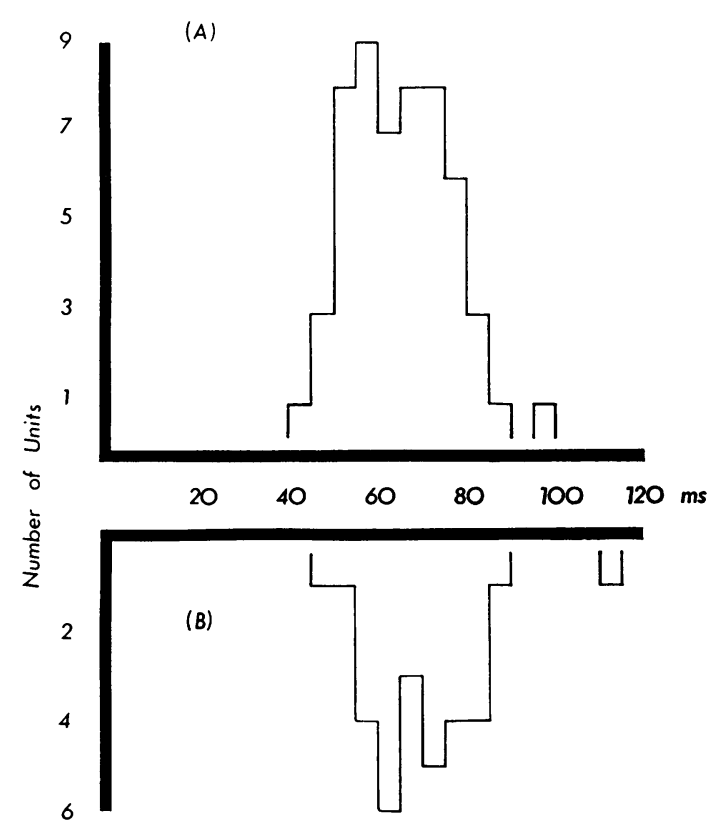

FIG. 6. Contraction times of threshold motor units. (A) Histogram of 55 threshold twitch contractions in six subjects using surface stimulation. (B) Histogram of an additional 30 threshold twitch contractions in two subjects using intramuscular stimulation.

tribution of contraction times found with percutaneous stimulation, a bipolar concentric needle electrode (Disa 9013K0512) was used as stimulating electrode in two subjects. This needle electrode was introduced subcutaneously, traversing the motor point in a number of parallel tracks, with threshold stimulation performed at a number of sites along each track. In this way 15 threshold contractions were recorded in each of the two subjects, as illustrated in Fig. 5b. The contraction times of these units are graphed in histogram form in Fig. 6b, and a unimodal distribution is again seen with a spectrum of contraction times very similar to that obtained with percutaneous stimulation.

The response of threshold motor units to prolonged tetanization at $50 \mathrm{~Hz}$ was studied in two subjects. In both subjects liminal stimulation of the ulnar nerve at the wrist and similar stimulation of the motor point via the bipolar needle electrode were used. If the threshold unit at a particular site was first identified using single

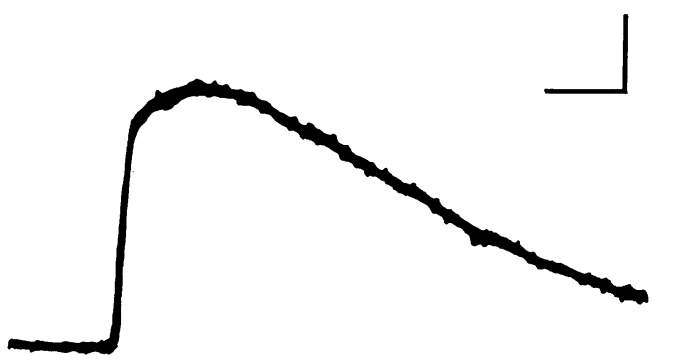

FIG. 7. Threshold tetanization at the motor point using intramuscular stimulation. Ten seconds after the oscilloscope sweep starts a small increment in stimulus voltage produces a threshold tetanic contraction which fatigues rapidly to $20 \%$ by $33 \mathrm{~s}$. In earlier oscilloscope sweeps, which are not illustrated, three small step increments at $10 \mathrm{~s}$ intervals did not produce contraction. Calibrations: vertical: $4.66 \times 10^{-} 5 \mathrm{Nm}$; horizontal: $5 s$.

shock stimulation, it was frequently found that tetanic stimulation at the same voltage level recruited additional units, often after some delay, during the course of an established tetanic contraction. Commonly the activation of the additional unit was at first inconstant so that an oscillating contraction appeared on top of the previously established tetanic level. As recruitment became consistent, the torque produced by the tetanic contraction rose to be level with the peak of the cyclical oscillations. Similarly, failure of activation of a unit was seen as an abrupt step down in tetanic torque or as a similar step preceded by cyclical oscillations. Thus the recruitment of an additional unit or the failure of activation of a motor unit produced characteristic changes in torque which could be readily recognized. The technique adopted consisted of continuous stimulation at $50 \mathrm{~Hz}$, initially at subthreshold levels, with small step-wise increments in stimulus voltage every $10 \mathrm{~s}$ until a threshold tetanic contraction was produced. In this way, it was possible to maintain the threshold tetanic contraction for some minutes without recruiting additional units. Nine threshold tetanic contractions have been studied. In eight, the level of tetanic torque fatigued within $60-90$ s to $14-20 \%$ of the peak level (Fig. 7). In one, torque decreased to $75 \%$ by $60 \mathrm{~s}$ and to $50 \%$ after five minutes. 


\section{DISCUSSION}

As demonstrated in this paper, ADM can be functionally isolated from other muscles of the hand so that its isometric responses can be readily studied, but it is not possible to correlate accurately mechanical properties with the surface EMG response, since this latter does not reflect activity of ADM alone. The results obtained appear quite compatible with those reported for other intrinsic muscles of the hand. Stimulation at the motor point at threshold appears to be a reasonable method for obtaining single motor unit twitches provided that adequate care is taken to minimize the difficulties detailed earlier. It must be conceded that electrical stimulation is an artificial method of activation compared with voluntary contraction, as has been used by Milner-Brown et al. (1973a, b, c), but the two techniques are essentially complementary, since each provides data not obtainable with the other.

In the cat a number of physiological parameters-namely, twitch contraction time, tetanic fusion frequency, and sensitivity to fatigue during prolonged tetanization-have been used to differentiate motor unit types and to correlate physiological type with histochemical profile (Burke et al., 1971). This paper represents an attempt to extend such studies to man. The response of ADM to prolonged tetanization suggests that at least some of the motor units of ADM are not resistant to fatigue. The form of prolonged tetanus used was presumably a more exhausting form of activation than that used in the cat by Burke et al. (1971) who tetanized individual motor units at $40 \mathrm{~Hz}$ for only $330 \mathrm{~ms}$ out of every second. Fatigue-resistant motor units were defined by these authors as those which maintained their tetanic tension in excess of $75 \%$ of the initial level after tetanization for two minutes. Fast-twitch, fatigue-resistant units ultimately fatigued, but only with further tetanization. Slow-twitch units did not fatigue even with prolonged activation, while fasttwitch, fatigue-sensitive units fatigued rapidly after activation for approximately $60 \mathrm{~s}$. That the degree of fatigue reported for ADM in the present study was not wholly attributable to failure of neuromuscular transmission is indicated by the initial lack of decrement in the evoked muscle action potential. Presumably, such mechanical fatigue as was recorded at this stage resulted from intrinsic muscular mechanisms, either from failure of excitation-coupling or of the contractile mechanism itself.

The unimodal distribution of threshold motor unit contraction times suggests that human ADM contains a homogeneous population of motor units. On the basis of contraction times, Sica and McComas (1971) have suggested that human EHB contains two populations of motor units, but it would be unlikely if these findings in a muscle of the foot could be applied to an intrinsic muscle of the hand which must perform different motor functions. Certainly in various upper and lower limb muscles of man, Buchthal and Schmalbruch (1970) have reported unimodal distributions of contractions times, there being no evidence of different muscle fibre groups with distinctive contraction times, and more recently Milner-Brown et al. (1973b) reported a normat distribution of contraction times for IDI, therg being no clear distinction between fast and slowe twitch units. However using the myofibrillat ATP-ase reaction, which in the cat reflects the physiological difference between fast and slow twitch fibres, Johnson et al. (1973) have diss. $\overrightarrow{0}$ tinguished two populations of muscle fibres it $\rightarrow$ human ADM, each comprising approximately $50 \%$ of the sample. The apparent discrepancy between histochemical properties reported by Johnson et al. (1973) and the physiological properties documented in the present study is unexplained. Nevertheless, Johnson et al. (1973) also reported two histochemically distinguishable groups of muscle fibres in those muscles studied physiologically by Buchthal and Schmalbruch (1970) and by Milner-Brown et al. (1973b) and found by these authors to have unimodal distributions of contraction times. The range of contraction times reported here for ADM, 40$100 \mathrm{~ms}$, agrees well with the $30-100 \mathrm{~ms}$ range reported by Milner-Brown et al. (1973b) for IDI, another ulnar-innervated intrinsic muscle of the hand.

The results of tetanic stimulation using thresh- N old stimulus levels suggest that at least some of $N$ the motor units in ADM conform with the fast- N్ contracting, rapidly-fatiguing group described in the cat by Burke et al. (1971), but it also seems 
likely that some units are fatigue-resistant. The effect of recruitment or drop-out of additional units was abrupt and readily recognized so that the progressive decline in tetanic contraction cannot be attributed to electrode displacement with resulting failure to activate a particular motor unit. It can be argued that threshold tetanic stimulation may activate more than one motor unit, but this objection can be raised to all studies involving threshold stimulation, and even were this so the progressive decrease in tetanic torque indicates that the involved motor unit or units was or were undergoing gradual contractile failure.

Thus the results of this study indicate that the motor unit population of ADM is homogeneous with respect to contraction time, and comprises fatigue-sensitive units and some units which, while relatively fatigue-resistant, do undergo some contractile failure with prolonged activation. These parameters are consistent with type FF and type FR motor units as described by Burke et al. (1971). Is there any necessity to postulate the presence in ADM of slow contracting non-fatiguing motor units (type $\mathrm{S}$ of Burke et al.)? At face value, the longer contraction times reported here would be consistent with the presence of slow-twitch units especially if human contraction times are directly comparable with those of the cat. But such is not the case. In the cat contractile force is measured directly, but values obtained in intact man reflect the inertia of non-contractile tissues. The torque recorded depends on the development of force at a joint which is moved by the appropriate muscle and so represents only an indirect approximation of contractile force. Thus a systematic distortion of the contraction time is introduced in the human subject by the recording technique. By relating the range of threshold motor unit contraction times to the total muscle contraction time it can be appreciated that at least half the population of threshold units have contraction times longer than those of the total muscle. It seems unlikely, therefore, that motor units with slower contraction times have been systematically neglected by the technique of identification and recording. It may therefore be concluded that if slow-twitch units exist in ADM they form one end of a normal spectrum of motor units and do not constitute a functionally distinct group.
The authors would like to acknowledge the constructive advice given by Professor J. W. Lance. Illustrations were photographed by the Department of Medical Illustration, University of New South Wales.

\section{REFERENCES}

Bergmans, J. (1970). The Physiology of Single Human Nerve Fibres. Vander: Louvain.

Brust, M., and Cosla, H. W. (1967). Contractility of isolated human skeletal muscle. Archives of Physical Medicine and Rehabilitation, 48, 543-555.

Buchthal, F., and Schmalbruch, H. (1970). Contraction times and fibre types in intact human muscle. Acta Physiologica Scandinavica, 79, 435-452.

Brown, W. F. (1972). A method for estimating the number of motor units in thenar muscles and the changes in motor unit count with ageing. Journal of Neurology, Neurosurgery, and Psychiatry, 35, 845-852.

Brown, W. F. (1973). Thenar motor unit count estimates in the carpal tunnel syndrome. Journal of Neurology, Neurosurgery, and Psychiatry, 36, 194-198.

Burke, D., Skuse, N. F., and Lethlean, A. K. (1974). The contractile properties of the abductor digiti minimi muscle in paramyotonia congenita. Journal of Neurology, Neurosurgery, and Psychiatry. (In press.)

Burke, R. E., Levine, D. N., Zajac, F. E., III, Tsairis, P., and Engel, W. K. (1971). Mammalian motor units: physiological-histochemical correlation in three types in cat gastrocnemius. Science, 174, 709-712.

Desmedt, J. E. (1958). Méthodes d'étude de la fonction neuromusculaire chez l'homme. Acta Neurologica et Psychiatrica Belgica, 58, 977-1017.

Desmedt, J. E., Emeryk, B., Hainaut, K., Reinhold, H., and Borenstein, S. (1973). Muscular dystrophy and myasthenia gravis. In New Developments in Electromyography and Clinical Neurophysiology, Vol. 1. pp. 380-399. Edited by J. E. Desmedt, Karger: Basel.

Desmedt, J. E., and Hainaut, K. (1968). Kinetics of myofilament activation in potentiated contraction: staircase phenomenon in human skeletal muscle. Nature, 217, 529532.

Eberstein, A., and Goodgold, J. (1968). Slow and fast twitch fibers in human skeletal muscle. American Journal of Physiology, 215, 535-541.

Johnson, M. A., Polgar, J., Weightman, D., and Appleton, D. (1973). Data on the distribution of fibre types in thirtysix human muscles. An autopsy study. Journal of the Neurological Sciences, 18, 111-129.

McComas, A. J., Fawcett, P. R. W., Campbell, M. J., and Sica, R. E. P. (1971). Electrophysiological estimation of the number of motor units within a human muscle. Journal of Neurology, Neurosurgery, and Psychiatry, 34, 121-131.

McComas, A. J., and Thomas, H. C. (1968). Fast and slow twitch muscles in man. Journal of the Neurological Sciences, 7, 301-307.

Merton, P. A. (1954). Voluntary strength and fatigue. Journal of Physiology, 123, 553-564.

Milner-Brown, H. S., Stein, R. B., and Yemm, R. (1973a). The contractile properties of human motor units during voluntary isometric contractions. Journal of Physiology, 228, 285-306.

Milner-Brown, H. S., Stein, R. B., and Yemm, R. (1973b) The orderly recruitment of human motor units during 
voluntary isometric contractions. Journal of Physiology, 230, 359-370.

Milner-Brown, H. S., Stein, R. B., and Yemm, R. (1973c). Changes in firing rate of human motor units during linearly changing voluntary contractions. Journal of Physiology, 230, 371-390.

Sica, R. E. P., and McComas, A. J. (1971). Fast and slow twitch units in a human muscle. Journal of Neurology, $\underset{\mathbb{Q}}{\mathrm{Z}}$ Neurosurgery, and Psychiatry, 34, 113-120.

Slomić, A., Rosenfalck, A., and Buchthal, F. (1968). Elec- Of trical and mechanical responses of normal and myasthenic muscles with particular reference to the staircase phenomenon. Brain Research, 10, v-xii, 1-78. 